\title{
Construção de tecnologias em saúde sobre o manejo de corpos no contexto do Novo Coronavírus
}

\author{
Construction of health technologies on the management of bodies in the context of the New \\ Coronavirus \\ Construcción de tecnologías sanitarias sobre la gestión de organismos en el contexto del Nuevo \\ Coronavirus
}

Recebido: 03/03/2021 | Revisado: 09/03/2021 | Aceito: 18/03/2021 | Publicado: 23/03/2021

Nayara Fernanda Alves Moreira

ORCID: https://orcid.org/0000-0003-1094-7399 Universidade do Estado do Pará, Brasil E-mail: nfernandamoreira@gmail.com

Lauany Silva de Medeiros

ORCID: https://orcid.org/0000-0002-5683-6347 Universidade do Estado do Pará, Brasil E-mail: lauanymedeiiros@gmail.com

Michele Pinheiro Ferreira

ORCID: https://orcid.org/0000-0001-5316-9908 Universidade do Estado do Pará, Brasil

E-mail: michelepinheiroferreira@gmail.com

Karen Silva de Castro

ORCID: https://orcid.org/0000-0001-8463-0322 Universidade do Estado do Pará, Brasil

E-mail: silvakaren2021@gmail.com

Renata Campos de Sousa Borges

ORCID: https://orcid.org/0000-0002-7510-5582 Universidade do Estado do Pará, Brasil E-mail: renatasousa88@hotmail.com

Sâmara Cristina Souto de Almeida ORCID: https://orcid.org/0000-0003-0173-1748 Hospital Municipal de Marabá, Brasil E-mail: smarasouto@outlook.com Daniele Lima dos Anjos Reis

ORCID: https://orcid.org/0000-0002-8447-6828 Universidade do Estado do Pará, Brasil E-mail: anjo.daniele@hotmail.com

Jose Ronaldo Teixeira de Sousa Junior ORCID: https://orcid.org/0000-0002-8730-9304 Centro Universitário do Estado do Pará, Brasil E-mail: ronaldosousajr@gmail.com

Milena Coelho Fernandes Caldato ORCID: https://orcid.org/0000-0002-7077-8470 Universidade do Estado do Pará, Brasil E-mail: milenacaldato@hotmail.com

Ismaelino Mauro Nunes Magno ORCID: https://orcid.org/0000-0003-3323-1465 Centro Universitário do Pará, Brasil E-mail: mauromagno@cesupa.br

Genislaine Ferreira Pereira

ORCID: https://orcid.org/0000-0002-4565-0379 Prefeitura Municipal de Tucuruí, Brasil E-mail: genislaine_fp@ hotmail.com

\section{Resumo}

Relatar a construção de tecnologias em saúde voltadas para profissionais sobre a manipulação de corpos no contexto do novo coronavírus. Trata-se de um relato de experiência, de caráter descritivo, baseando-se a partir da análise e descrição sobre o desenvolvimento tecnologias em saúde, que visaram democratizar informações acerca dos cuidados com os casos suspeitos ou confirmados de Covid-19 que evoluíra ao óbito. O estudo foi fundamentado a partir da leitura do protocolo "Manejo de Corpos do Novo Coronavírus", disponibilizado pelo Ministério da Saúde do Brasil e ocorreu em 5 etapas: 1) Diagnóstico situacional; 2) Revisão bibliográfica; 3) Elaboração da proposta; 4) Construção 
das tecnologias e 5) Implementação da tecnologia. Logo, como principais resultados desta pesquisa ressalta-se a construção de três tecnologias em saúde: Uma em forma de cartilha de tamanho 33 por $19 \mathrm{~cm}$, distribuída para os profissionais, dois fluxogramas distribuídos nos hospitais e uma mídia em forma de vídeo de 1 min e 20s, compartilhado nas redes sociais e aplicativo multiplataforma de mensagens. Por fim, houve a implementação desses instrumentos, a partir da divulgação da mídia aos profissionais de saúde e comunidade profissional. Concluiu-se neste trabalho que vincular os dois aspectos de educação e saúde necessita de aptidão profissional para que as informações alcancem o público destinado, logo, as tecnologias propostas obtiveram efeito positivo na comunidade a partir de sua divulgação no meio digital. Ademais, destaca-se a necessidade da construção de tecnologias que contribuam para esclarecer e enfrentar, com menos danos, as novas epidemias mundiais.

Palavras-chave: Covid-19; Pandemia; Tecnologia em saúde.

\begin{abstract}
To report the construction of health technologies aimed at professionals about the manipulation of bodies in the context of the new coronavirus. This is an experience report, of a descriptive character, based on the analysis and description of the development of health technologies, which aimed to democratize information about care with suspected or confirmed cases of Covid-19 that had evolved to death. The study was based on the reading of the protocol "Body Management of the New Coronavirus", made available by the Ministry of Health of Brazil and occurred in 5 stages: 1) Situational diagnosis; 2) Literature review; 3) Elaboration of the proposal; 4) Construction of technologies and 5) Implementation of technology. Therefore, the main results of this research are the construction of three health technologies: One in the form of a booklet of size 33 by $19 \mathrm{~cm}$, distributed to professionals, two flowcharts distributed in hospitals and a media in the form of a $1 \mathrm{~min}$ video. 20s, shared on social networks and multiplatform messaging application. Finally, there was the implementation of these instruments, from the dissemination of the media to health professionals and the professional community. It was concluded in this work that linking the two aspects of education and health requires professional aptitude so that the information reaches the intended audience, therefore, the proposed technologies obtained a positive effect in the community from their dissemination in the digital environment. In addition, the need to build technologies that contribute to clarify and face, with less damage, the new global epidemics, is highlighted.
\end{abstract}

Keywords: Covid-19; Pandemic; Health technology.

\title{
Resumen
}

Informar la construcción de tecnologías sanitarias dirigidas a profesionales sobre la manipulación de cuerpos en el contexto del nuevo coronavirus. Se trata de un relato de experiencia, de carácter descriptivo, basado en el análisis y descripción del desarrollo de tecnologías sanitarias, que tuvo como objetivo democratizar la información sobre la atención con casos sospechosos o confirmados de Covid-19 que habían evolucionado hasta la muerte. El estudio se basó en la lectura del protocolo "Manejo Corporal del Nuevo Coronavirus", puesto a disposición por el Ministerio de Salud de Brasil y ocurrió en 5 etapas: 1) Diagnóstico situacional; 2. Revisión de la literatura; 3) Elaboración de la propuesta; 4) Construcción de tecnologías y 5) Implementación de tecnología. Por tanto, los principales resultados de esta investigación son la construcción de tres tecnologías sanitarias: una en forma de cuadernillo de tamaño 33 por 19 $\mathrm{cm}$, distribuida a profesionales, dos diagramas de flujo distribuidos en hospitales y un medio en forma de vídeo de $1 \mathrm{~min}$. 20s, compartido en redes sociales y aplicación de mensajería multiplataforma. Finalmente, estaba la implementación de estos instrumentos, desde la difusión de los medios de comunicación a los profesionales de la salud y la comunidad profesional. En este trabajo se concluyó que vincular los dos aspectos de la educación y la salud requiere de aptitud profesional para que la información llegue al público destinatario, por lo que las tecnologías propuestas obtuvieron un efecto positivo en la comunidad a partir de su difusión en el entorno digital. Además, se destaca la necesidad de construir tecnologías que contribuyan a esclarecer y enfrentar, con menos daño, las nuevas epidemias globales.

Palabras clave: Covid-19; Pandemia; Tecnología sanitaria.

\section{Introdução}

Em dezembro de 2019, a cidade de Wuhan, capital da província de Hubei, na China, tornou-se o centro de um surto de pneumonia de causa não conhecida, ligada epidemiologicamente ao mercado local de frutos do mar. Tal agente etiológico foi isolado e, posteriormente, conhecido como Sars-Cov-19 que é um vírus membro da família dos coronavírus, que é o causador de uma Síndrome Respiratória Aguda Grave (SRAG) (Catton et al, 2020).

Esta doença tornou-se uma preocupação mundial quando a Organização Mundial da Saúde (OMS) em 11 de março de 2020 em Genebra, na Suíça, constituiu o Covid-19 como pandemia, ou seja, uma emergência de saúde pública de importância internacional que já afeta todos os continentes. Nesse sentido, o coronavírus ganhou um novo status alerta, pois além dele 
causar complicações respiratórias graves, a sua fácil transmissão por gotículas e partículas aerossóis infectados, a não imunidade da maioria população, o pouco conhecimento sobre o vírus e o tratamento ineficaz para a doença torna a situação mais preocupante.

Levando isto em consideração, as medidas de isolamento social e quarentena são consideradas, atualmente, o melhor modo de diminuir o impacto da contaminação. Todavia, para os profissionais de saúde, especialmente aqueles considerados linha de frente contra o Covid-19 desde a atenção primária até a rede hospitalar estas recomendações não são possíveis de serem concebidas (Teixeira et al, 2020).

Outrossim, percebe-se a evolução contínua da taxa de mortalidade devido a essa infecção, haja vista que desde o primeiro óbito no dia 17 de março de 2020, o número de mortes cresceu gradativamente, atingindo cerca de 9 mil óbitos registrados após dois meses do conhecimento da doença. Além disso, índices retirados do Ministério da Saúde (MS) demonstram que a média de mortes no Brasil, a cada 7 dias, varia entre 900 a 1000 casos, sendo avaliado que essa taxa sofre acréscimos e modificações gradativas no número de casos confirmados evoluídos a óbitos, salientando-se que grande parte de casos são subnotificados, por isso, a estimativa é que existam ainda mais números de morte no país (França et al, 2020).

Nessa perspectiva, nota-se que a disseminação da doença se amplia cada vez mais, e com isso, surge a necessidade de criações de políticas que venham contribuir para a prevenção e consequente minimização do número de óbitos pela patologia. Dessa forma, observa-se a indispensabilidade de manejos específicos sobre como deve ser estabelecida a realização dos procedimentos e cuidados, para evitar a contaminação pela doença (Werneck \& Carvalho, 2020).

Isto posto, é perceptível que as equipes de saúde estão passando por uma por uma das maiores pressões físicas e psicológicas do século. Acima disso, estão sofrendo ainda risco de infecção, tendo que trabalhar com novos protocolos e equipamentos de proteção individual que mudam frequentemente e tornam o setor de saúde vulnerável ao adoecimento (Walton et al, 2020).

Logo, é importante ressaltar que estes protocolos mudam constantemente na pandemia e servem na área da saúde como uma espécie de diretrizes, instruções específicas a serem seguidas pelos profissionais com o objetivo de orientar decisões sobre critérios técnicos-científicos (Zwielewski et al, 2020).

Um desses protocolos utilizados é o "Protocolo de Manejo de corpos no contexto do novo coronavírus Covid-19" tal instrumento visa fornecer recomendações referentes ao manejo de corpos no contexto da Covid-19 e outras questões gerais acerca desses óbitos, haja a vista que manipulação dos corpos de forma inadequada ou a realização de necropsia pode acarretar em contaminação (Ministério da Saúde do Brasil, 2020).

Nesse interim, as tecnologias em saúde (TS) compõem um instrumento de extrema importância para o avanço do campo médico-cientifico, uma vez que constituem diversos instrumentos utilizados para otimizar e abranger o cuidado ao indivíduo de forma íntegra e eficaz. Além do mais, segundo a Organização Mundial da Saúde (OMS), a população mundial deve ser amplamente educada sobre as condições e complicações evidenciadas pelo Covid-19, de modo que essas tecnologias sirvam para a promoção da prevenção dessa enfermidade.

Ademais, visando qualificar os serviços de saúde prestados ao cuidado ao paciente, por meio das relações interpessoais e criação de vínculos com a equipe multiprofissional, além da melhoria dos instrumentos necessários para a promoção de saúde dos clientes, as tecnologias em saúde constituem-se em métodos essenciais para a minimização de efeitos e gravidade da enfermidade e prevenção da doença, contribuindo para a sensibilização da população e o enfrentamento à pandemia, de forma integra e abrangente (Schneider, 2020).

A vista disso, diante do cenário vigente de pandemia instalada, percebe-se que o uso de tecnologias educacionais é essencial para obter e explanar informações de forma acessível e esclarecedora sobre medidas de prevenção e cuidado contra o Covid-19, por esse motivo, a pesquisa teve o objetivo de relatar a construção de tecnologias em saúde voltadas para 
profissionais sobre a manipulação de corpos no contexto do novo coronavírus, buscando promover o ensino da conduta adequada sobre esse manejo.

\section{Metodologia}

O estudo trata-se de um relato de experiência, de caráter descritivo e qualitativo, baseando-se a partir da análise e descrição sobre o desenvolvimento tecnologias em saúde (TS), com o intuito de democratizar informações acerca dos cuidados com os casos suspeitos ou confirmados de Covid-19 que evoluíra ao óbito. Logo esta pesquisa, buscou uma abordagem facilitadora da aprendizagem, se constitui o corpus da metodologia ativa, como recomenda Pereira et al. (2018).

Por conseguinte, o estudo foi fundamentado a partir da leitura do protocolo "Manejo de Corpos do Novo Coronavírus", disponibilizado pelo Ministério da Saúde do Brasil (2020), contribuindo para a formulação do relato, que se dividiu em 4 etapas: 1) Diagnóstico situacional; 2) Revisão bibliográfica; 3) Elaboração da proposta; 4) Construção das tecnologias e 5) Implementação da tecnologia.

Na primeira etapa houve a percepção do diagnóstico situacional que ocorreu por meio de uma integração intersetorial entre a vigilância em saúde e o curso de graduação em enfermagem da universidade, devido a uma demanda local para fortalecer as ações de educação em saúde e educação permanente sobre o ovid-19.

Na segunda etapa, buscou-se formular o tema base por meio do estabelecimento de uma questão norteadora. A partir disso, seguiu-se para a coleta de dados por meio da revisão bibliográfica, onde buscou-se artigos que abordassem sobre a problemática, indexados nas plataformas de busca online Scielo, Biblioteca Virtual de Saúde (BVS) e Google Acadêmico por meio dos seguintes descritores "Tecnologias em Saúde", "Protocolo" e "Covid-19". Tal busca, resultou em 20 artigos.

Em sequência, houve a elaboração da proposta, em que se recorreu ao emprego da educação em saúde, por meio da confecção e divulgação de mídias educativas pelas redes sociais, como método de orientação segura e facilitadora para a população, visto que as atividades presenciais tornar-se-iam inviáveis devido o período vigente de pandemia instalada.

Ademais, seguiu-se para a construção das tecnologias que foi realizada no Microsoft Power Point versão 2016, em que foram produzidas uma cartilha de tamanho 33 por $19 \mathrm{~cm}$ (Figura 1), distribuída para os profissionais, dois fluxogramas distribuídos nos hospitais (Figuras 2 e 3) e uma mídia em forma de vídeo de 1min e 20s (Figura 4), compartilhado nas redes sociais e aplicativo multiplataforma de mensagens. Por fim, houve a implementação desses instrumentos, a partir da divulgação da mídia aos profissionais de saúde e comunidade profissional.

Nesse sentido, torna-se necessário que se perceba por meio das imagens, as quais buscaram ofertar em todos as tecnologias expostas, meios didáticos e ilustrativos que diminuíssem a rigidez do conteúdo proposto, haja vista que o tema especifico retrata aspectos relacionados aos óbitos, o qual é um ponto que pode afligir o psicológico dos profissionais. Contudo, ao mesmo tempo as ferramentas elaboradas frisaram a importância e seriedade do assunto. 
Research, Society and Development, v. 10, n. 3, e50610313488, 2021

(CC BY 4.0) | ISSN 2525-3409 | DOI: http://dx.doi.org/10.33448/rsd-v10i3.13488

Figura 1: Cartilha voltada aos profissionais de saúde acerca do manejo de corpos no contexto do novo coronavírus.

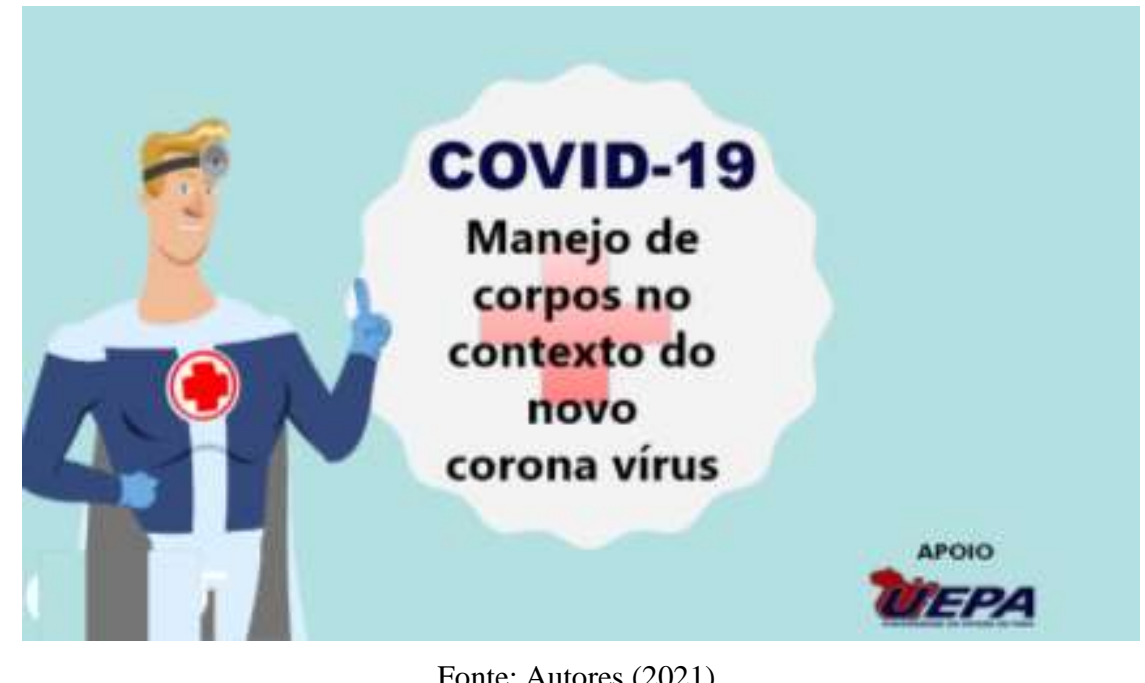

Figuras 2 e 3: Fluxogramas produzidos e destinados ao setor hospitalar acerca do manejo de corpos no ambiente domiciliar e hospitalar contexto do novo coronavírus.

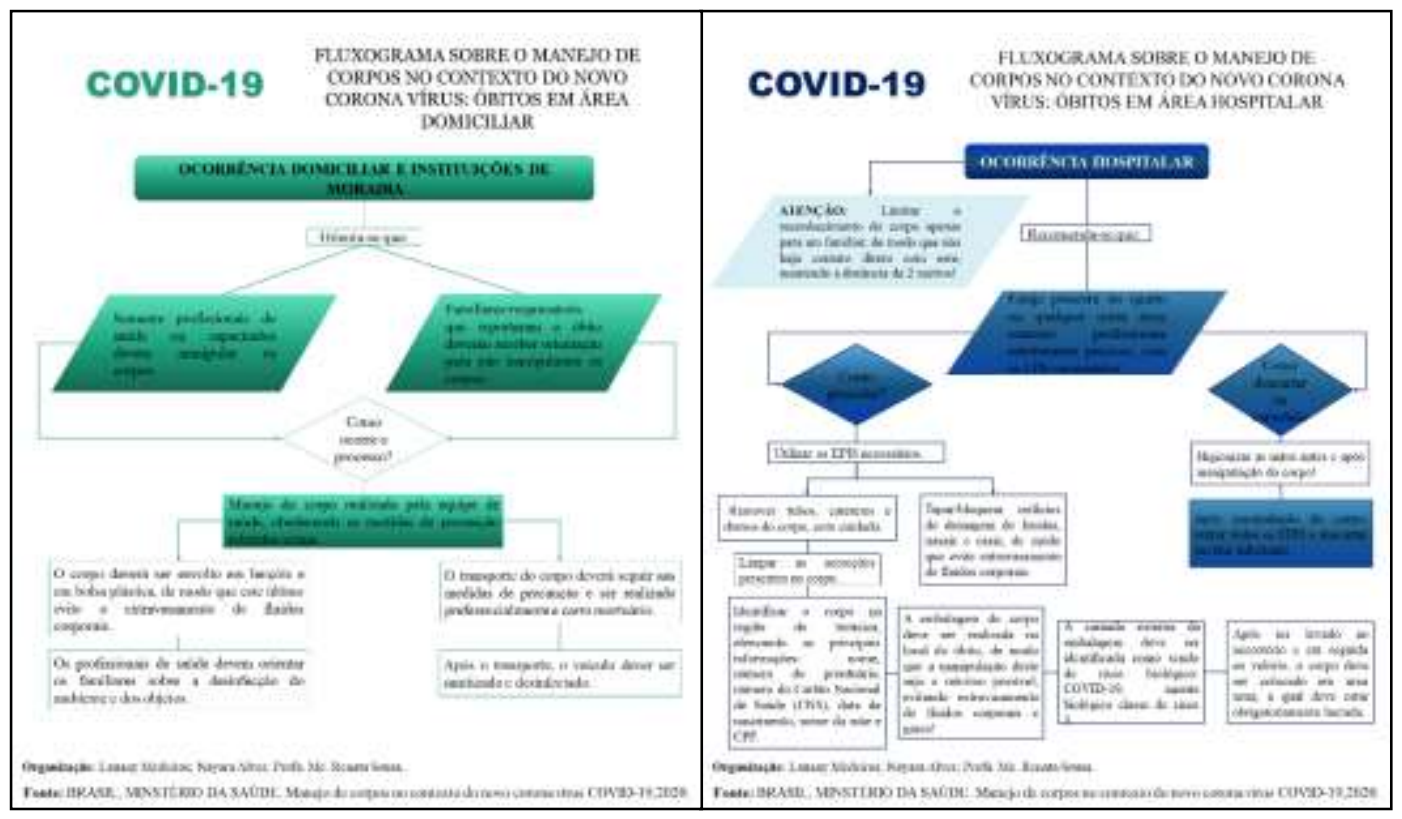

Fonte: Autores (2021). 
Figura 4: Arquivo em mídia produzido acerca dos cuidados no manejo de corpos contexto do novo coronavírus.

\section{CORONAVÍRUS}

\section{A T E N Ç Â O}

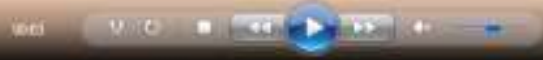

Fonte: Autores (2021).

\section{Resultados e Discussão}

O combate ao coronavírus necessita de uma série de esforços de distintos setores, atores e instituições da sociedade. Logo, por a pandemia ser um problema de saúde coletivo com escasso arcabouço tecnológico e científico disponível na atualidade, o apoio de tecnologias educacionais de cunho virtual com embasamento teórico voltada aos profissionais de saúde é essencial (Schneider, 2020). Pois segundo De Medeiros et al, (2020):

(...) o uso desse método corrobora para o desenvolvimento ou exploração de recursos intelectuais, principalmente, voltado a educação em saúde, durante a pandemia da Covid-19. Desse modo, mesmo de longe a sociedade permanece sendo informada e conscientizada sobre a importância de estimular o bem-estar físico, psicológico, espiritual e emocional, apesar da medida preventiva de quarentena (De Medeiros et al, p. 4, 2020)

Nesse sentido, tais tecnologias buscaram educar os profissionais de saúde, em especial os da linha de frente do combate ao SARS-CoV-2, e a população em geral com uma linguagem acessível e adaptada a todos os públicos de interesse, com meios circulação simples, dentre eles mídias sociais, grupos de conversa, plataformas de acesso em massa que, segundo Neto et al, (2020) facilitam o processo tanto de educação em saúde, como o de educação permanente:

As tecnologias e o pensar científico nunca estiveram tão associados nesta nova perspectiva e necessidade de ensinar em tempos de reclusão. Talvez seja um dos aspectos positivos a ser herdado pós-pandemia: a reconexão e reafirmação do pensar e os avanços da ciência e da informação, juntas e indissociável, através de uma interrelação entre a saúde e educação. (Neto et al. p. 5, 2020)

No que tange as limitações encontradas durante a construção das TS, foi possível perceber a escassez na literatura sobre o tema, assim como a inexistência de outras tecnologias acerca do novo coronavírus. Desse modo, utilizou-se o protocolo supracitado para complementação do estudo, de maneira que as TS evidenciassem o papel dos profissionais frente ao manejo e 
controle da infecção, bem como instrumentos necessários para oferecer o embasamento teórico critico-reflexivo para a sua proteção contra o Sar-Cov-19 e a sua qualificação para o enfrentamento desta patologia.

Isto posto, a fim de avaliar o protocolo e se as tecnologias propostas foram eficazes, o trabalho precisou ser avaliado por três aspectos principais: $1^{\circ}$ ) Uma análise do protocolo para subsídio teórico; $2^{\circ}$ ) A utilização de tecnologias no setor de saúde $3^{\circ}$ ) Principais dificuldades para implementação e a experiência de acadêmicos enfermagem frente às pesquisas (Santos, et al, 2016).

\section{Uma análise do protocolo para subsídio teórico}

Segundo o dicionário Aurélio (2020) protocolos podem ser definidos, de um modo geral, como um conjunto de normas, regras ou orientações sobre como realizar uma intervenção profissional que se deve respeitar. No âmbito da saúde, de acordo com Zwielewski et al. (2020) protocolos considerados diretrizes, instruções e/ou procedimentos singulares que necessitam serem adotados por profissionais com finalidade de conduzir decisões ou procedimentos com critérios técnicoscientíficos.

Nesse sentido, tendo em vista a pandemia instalada do Covid-19, no início do ano de 2020, foram formuladas iniciativas que previssem a prevenção e controle dos casos da doença. Nesse sentido, segundo o relatório da Organização Mundial da Saúde (OMS), em junho desse ano, o número de casos confirmados aumentou relativamente, atingindo cerca de 7,8 milhões de pessoas confirmadas, dado esse que progressivamente se maximizou com o decorrer dos meses.

Mediante isso, por se tratar de uma patologia altamente infectocontagiosa, tecnologias foram formuladas, dando ênfase a estruturação de protocolos, normas e sistematização dos setores hospitalares e serviços de saúde, para a efetivação da segurança populacional e qualidade de assistência profissional prestada. Desse modo, métodos de prevenção e segurança tornam-se fatores essenciais para a minimização da cadeia de contagio.

Destarte, visando a segurança dos profissionais de saúde que estão mais expostos às situações de risco diariamente, por atuarem na linha de frente do combate ao SARS-CoV-2, foi criado o Protocolo de Manejo de Corpos no Contexto do Novo Coronavírus, pelo Ministério da Saúde (2020), o qual tem como objetivo, demandar as normas instituídas para o preparo do corpo, visando a segurança do profissional responsável pela prática, evitando o contato deste com a exposição a sangue e fluidos corporais infectados, além de objetivos ou outras superfícies contaminadas.

Este protocolo é divido em seis tópicos que explanam desde recomendações a óbitos que ocorrem em ambiente hospitalar, a necropsia e equipamentos de proteção individual voltado aos profissionais da saúde, até mortes que ocorrem em ambiente domiciliar, instituições de moradia e ambientes públicos direcionados a abordagem familiar. Tal estrutura, segundo Silva et al, (2020), melhora a assistência e a segurança dos profissionais e da população em geral.

Infere-se, portanto, que os protocolos são um recurso fundamental para o combate da pandemia de Covid-19, pois além de padronizar o atendimento promovem a segurança tanto do paciente como a do profissional de saúde. Contudo tais informações necessitam de dispositivos que facilitem a divulgação afim de preparar profissionais para o enfretamento do novo coronavírus

\section{A utilização de tecnologias no setor de saúde}

A primeira etapa caracterizou-se pela análise e descrição do protocolo, necessário para a constatação sobre o manejo de corpos no contexto do novo coronavírus, bem como a adequação do conteúdo ao público referenciado. A partir disso, foi realizado a segunda etapa, referente a estruturação do assunto, objetivando elencar, destacar e demonstrar a temática correta sobre a problemática. 
Outrossim, considera-se a importância de tecnologias em saúde para a formulação de processos e produtos que visem o envolvimento e o desenvolvimento do vínculo entre os profissionais de saúde e seus clientes, para a promoção de uma educação em saúde íntegra e eficaz, que permita a explanação de informações relevantes ao público-alvo de forma acessível e facilitadora (Nietsche et al, 2014).

Nessa perspectiva, mediante a observação de que não há uma estratégia profilática para a contenção da alta taxa de transmissibilidade do Covid-19, elencam-se a utilização de tecnologias de saúde educativas para a promoção de medidas preventivas que auxiliem na sensibilização da população sobre a patologia e como evitá-la, por meio de diversas metodologias educativas em que os profissionais de saúde possam abordar amplamente sobre a educação em saúde sobre o Covid-19. Além disso, segundo Palácio \& Takenami (2020), a educação em saúde corresponde a um processo político pedagógico.

Dessa forma, observa-se que a educação em saúde é imprescindível para a conscientização da população em todo o mundo. Entretanto, os meios pelos quais elas são explanadas tornam-se um desafio mediante a observação de que não se lidará somente com um público em questão, sendo assim, esses instrumentos devem ser acessíveis a todos, e contribuírem não somente para a percepção da comunidade, mas também para a construção de saberes dos profissionais (De Jesus Martins \& De Albuquerque 2007).

Nesse sentido, as tecnologias elaboradas buscaram atender ao público-alvo estipulado, de forma didática e esclarecedora sobre a manipulação de corpos no contexto do novo coronavírus, contribuindo para a formação dos acadêmicos e principalmente para a elucidação da abordagem correta sobre a temática, permeando a importância das tecnologias de informação para a prática de saúde e contribuindo para a educação preventiva sobre o Covid-19.

\section{Principais dificuldades para implementação frente às pesquisas}

A propagação do vírus em todo o mundo, nota-se que informações preventivas são essenciais para a manutenção da saúde, nesse contexto, a construção e implementação de tecnologias voltadas a divulgação de informações fidedignas são essenciais para a comunidade, além disso acrescentam conhecimento que contribuíram futuramente para a formação de profissionais competentes (Neto et al, 2020).

Contudo, tais ações necessitam ser cuidadosamente construídas levando em consideração o desconhecimento das particularidades do novo coronavírus. Além disso, para promover educação em saúde ou a educação permanente para a população e profissionais da saúde têm que se avaliar as peculiaridades do público alvo, como idade, diversidade, pluralidade e a complexidade do indivíduo, como recomenda Mallmann et al. (2015).

Isto posto, outro empecilho já no que tange a divulgação das tecnologias é a resistência do recebimento das informações, haja a vista que ao mesmo tempo que estas novas tecnologias educacionais representam oportunidades de aprendizado, elas podem se tornar um obstáculo quando mal utilizadas, como no caso das Fake News. Tal dificuldade também foi encontrada no estudo de Palácio e Takenami, (2020)

Amplamente divulgadas, as recomendações dos órgãos de saúde para conter o avanço da epidemia no Brasil têm enfrentado resistência e revelado muitos limites e desafios para profissionais de saúde, sobretudo em relação às práticas de educação em saúde. Essas ainda se encontram fortemente marcadas por concepções tradicionais e verticalizadas, e têm se revelado de forma pontual e fragmentada nas ações de assistência e vigilância à saúde. Ademais, a massiva veiculação de informações falsas ou divergentes daqueles oficiais tem comprometido a adesão da população às recomendações de prevenção à Covid-19. (Palácio e Takenami, p. 11, 2020) 
Isto posto, o último obstáculo encontrado referiu-se ao meio de divulgação das tecnologias que ocorreram em meio a crise que acarretou a pandemia e ao isolamento social, dessa forma, teve-se que se utilizar mídias online para a divulgação das tecnologias, averiguando-se que cada vez mais as inovações tecnológicas estão incorporadas no cotidiano das pessoas.

\section{Conclusão}

Destarte, foi elucidado que a construção de TS, tem potencial para possibilitar o acesso à informação de forma rápida e sintetizada aos profissionais de saúde, de forma que possam interferir na cadeia de transmissão do vírus e na proteção dos trabalhadores linha de frente.

Entretanto, vincular os dois aspectos de educação e saúde necessita de aptidão profissional para que as informações alcancem o público destinado. Sendo que ao mesmo tempo que estas novas metodologias educacionais representam oportunidades de aprendizado, elas podem se tornar um obstáculo quando mal utilizadas, como no caso das Fake News.

Logo, as tecnologias propostas obtiveram efeito positivo na comunidade a partir de sua divulgação no meio digital. E se repassada de forma dinâmica e clara para os profissionais de saúde, abrange um número crescente de pessoas a virem ser laureadas por tal. Ademais, destaca-se a necessidade da construção de tecnologias que contribuam para esclarecer e enfrentar, com menos danos possíveis as novas epidemias mundiais, nesse sentido, recomendam-se a criação de novos estudos e tecnologias acerca das precauções por Covid-19 em todos os âmbitos.

\section{Referências}

Caetano, R., Silva, A. B., Guedes, A. C. C. M., Paiva, C. C. N. D., Ribeiro, G. D. R., Santos, D. L., \& Silva, R. M. D. (2020). Desafios e oportunidades para telessaúde em tempos da pandemia pela Covid-19: uma reflexão sobre os espaços e iniciativas no contexto brasileiro. Cadernos de Saúde Pública, 36 , e00088920.

Casas, C. P. R., Silva, J., Castro, R., Ribeiro-Alves, M., \& Franco, C. M. (2020). Avaliação de tecnologias em saúde: tensões metodológicas durante a pandemia de Covid-19. Estudos Avançados, 34(99), 77-96.

Catton, H (2020). Enfermagem na pandemia COVID - 19 e além: protegendo, salvando, apoiando e homenageando os enfermeiros. Revista Internacional de Enfermagem, 67(2), 157-159.

Ceccon, R. F. \& Schneider, I. J. C. (2020). Tecnologias leves e educação em saúde no enfrentamento à pandemia da Covid-19. SciELO Public Health. 1-19.

Coelho, A. L., de Araujo Morais, I., \& da Silva Rosa, W. V. (2020). A utilização de tecnologias da informação em saúde para o enfrentamento da pandemia do Covid-19 no Brasil. Cadernos Ibero-Americanos de Direito Sanitário, 9(3), 183-199.

De Almeida Hammerschmidt, K. S., \& Santana, R. F. (2020). Saúde do idoso em tempos de pandemia Covid-19. Cogitare enfermagem, 25.

De Corpos, R. A. D. M. (2020) Nota Técnica Covid-19 N 50/2020-SESA/SSAS/SSVS Recomendações acerca do manejo de corpos no contexto da pandemia da Covid-19. Ministério da saúde.

De Jesus Martins, J. \& de Albuquerque, G. L. (2007). A utilização de tecnologias relacionais como estratégia para humanização do processo de trabalho de saúde. Ciência, Cuidado e Saúde, 6(3), 351-356.

De Medeiros, L. S., de Castro, K. S., de Moura, P. G. S., Ferreira, M. P., Medeiros, T. D. S. P., \& da Silva, N. K. N. (2020). Analise epidemiologica descritiva nos primeiros 30 dias de casos confirmados de Covid-19 na Amazônia legal brasileira. Brazilian Journal of Health Review, 3(3), $4906-4928$.

França, E. B., Ishitani, L. H., Teixeira, R. A., Abreu, D. M. X. D., Corrêa, P. R. L., Marinho, F., \& Vasconcelos, A. M. N. (2020). Óbitos por Covid-19 no Brasil: quantos e quais estamos identificando? Revista Brasileira de Epidemiologia, 23, e200053.

Mallmann, D. G., Galindo Neto, N. M., Sousa, J. D. C., \& Vasconcelos, E. M. R. D. (2015). Educação em saúde como principal alternativa para promover a saúde do idoso. Ciência \& Saúde Coletiva, 20, 1763-1772.

Ministério da Saúde (BR). Secretaria de atenção especializada à saúde (2020). Protocolo de manejo clínico da Covid19 na atenção especializada. $1^{\text {a }}$ ed. Brasília: Ministério da Saúde.

Neto, J. B. D. S. B., de Castro, T. M. G., de Sousa Borges, R. C., dos Anjos Reis, D. L., Medeiros, T. D. S. P., Caldato, M. C. F., \& Magno, I. M. N. (2020). Construção de tecnologias educativas como forma de educação em saúde para a prevenção da Covid-19: relato de experiência. Revista Eletrônica Acervo Saúde, 12(9), e3737-e3737.

Neto, J. M. A. (2020). Sobre ensino, aprendizagem e a sociedade da tecnologia: por que se refletir em tempo de pandemia? Prospectus, 2(1), 28-38. 
Research, Society and Development, v. 10, n. 3, e50610313488, 2021

(CC BY 4.0) | ISSN 2525-3409 | DOI: http://dx.doi.org/10.33448/rsd-v10i3.13488

Nietsche, E. A., Teixeira, E., \& Medeiros, H. P. (2014). Tecnologias cuidativo-educacionais: uma possibilidade para o empoderamento do (a) enfermeiro (a). Porto Alegre: Moriá.

Palácio, M. A. V., \& Takenami, I. (2020). Em tempos de pandemia pela Covid-19: o desafio para a educação em saúde. Vigilância Sanitária em Debate: Sociedade, Ciência \& Tecnologia (Health Surveillance under Debate: Society, Science \& Technology), 8(2), 10-15.

Pereira A.S. et al. (2018). Metodologia da pesquisa científica.. UFMS

Santos, J. S., \& Teixeira, C. F. (2016). Política de saúde no Brasil: produção científica 1988-2014. Saúde em Debate, 40(108), 219-230.

Silva, A. S. T. D., Pinto, R. L. G., \& Martins, A. A. (2020). Implantação do protocolo de manejo de corpos pós-óbito no contexto do novo Coronavírus. J. nurs. health, 20104013-20104013.

Souza, T. S. D., \& Miranda, M. B. S. (2017). Horticultura como tecnologia de saúde mental.

Teixeira, C. F. D. S., Soares, C. M., Souza, E. A., Lisboa, E. S., Pinto, I. C. D. M., Andrade, L. R. D., \& Espiridião, M. A. (2020). A saúde dos profissionais de saúde no enfrentamento da pandemia de Covid-19. Ciência \& Saúde Coletiva, 25, 3465-3474.

Walton, M., Murray, E., \& Christian, M. D. (2020). Mental health care for medical staff and affiliated healthcare workers during the Covid-19 pandemic. European Heart Journal: Acute Cardiovascular Care, 9(3), 241-247.

Werneck, G. L., \& Carvalho, M. S. (2020). A pandemia de Covid-19 no Brasil: crônica de uma crise sanitária anunciada. Cad. Saúde Pública 36 (5) 8 Maio 20202020

World Health Organization. Prevenção e controle de infecção durante os cuidados de saúde quando houver suspeita de nova infecção por coronavírus (nCoV). OMS, 2020. Disponível em: <https://www.who.int/publications-detail/infection-prevention-and-control-during-health-care-when-novel-coronavirus-(ncov)infection-is-suspected-20200125>

Zwielewski, G.; Oltramari, G; Santos, A. R. S., Nicolazzi, E. D. S., Moura, J. D., Sant'Ana, V. L. P; \& Cruz, R. (2020). Protocolos para tratamento psicológico em pandemias: as demandas em saúde mental produzidas pela Covid-19. Rev debates in psychiatry-Ahead of print. 